\title{
Panspecies molecular assays detect viral pathogens missed by real-time PCR/ reverse-transcriptase PCR among pneumonia patients, Sarawak, Malaysia
}

Jane K. Fieldhouse 1,2,3, Emily S. Bailey ${ }^{1,2,4}$, Teck-Hock Toh ${ }^{5,6,7}$, King-Ching Hii ${ }^{8}$, Kerry A. Mallinson², Jakie Ting ${ }^{5,6}$, John A. Lednicky ${ }^{9,10}$, Antoinette Berita ${ }^{8}$, Tham Thi Nguyen ${ }^{11}$, Diego Galan ${ }^{1,11}$, Son T. Than ${ }^{11}$, See-Chang Wong ${ }^{6,7}$, Toh-Mee Wong ${ }^{5,6,12}$, Patrick J. Blair ${ }^{13}$ and Gregory C. Gray ${ }^{1,2,11,14^{*}}$ (D)

\begin{abstract}
Background: In a year-long pneumonia etiology study conducted June 2017 to May 2018 in Sarawak, Malaysia, 599 patients' nasopharyngeal swab specimens were studied with real-time polymerase chain reaction (rPCR)/ reversetranscription (rRT-PCR) assays for respiratory pathogens known to contribute to the high burden of lower respiratory tract infections. The study team sought to compare real-time assay results with panspecies conventional molecular diagnostics to compare sensitivities and learn if novel viruses had been missed.

Methods: Specimens were studied for evidence of adenovirus (AdV), enterovirus (EV) and coronavirus (CoV) with panspecies gel-based nested PCR/RT-PCR assays. Gene sequences of specimens positive by panspecies assays were sequenced and studied with the NCBI Basic Local Alignment Search Tool software.

Results: There was considerable discordance between real-time and conventional molecular methods. The realtime AdV assay found a positivity of 10.4\%; however, the AdV panspecies assay detected a positivity of $12.4 \%$ and the conventional AdV-Hexon assay detected a positivity of 19.6\%. The CoV and EV panspecies assays similarly detected more positive specimens than the real-time assays, with a positivity of $7.8 \%$ by the CoV panspecies assay versus $4.2 \%$ by rRT-PCR, and $8.0 \%$ by the EV panspecies assay versus $1.0 \%$ by rRT-PCR. We were not able to ascertain virus viability in this setting. While most discordance was likely due to assay sensitivity for previously described human viruses, two novel, possible zoonotic AdV were detected.
\end{abstract}

Conclusions: The observed differences in the two modes of amplification suggest that where a problem with sensitivity is suspected, real-time assay results might be supplemented with panspecies conventional PCR/RT-PCR assays.

Keywords: Pneumonia, Respiratory viruses, Molecular assay, Panspecies, Malaysia

\footnotetext{
* Correspondence: gregory.gray@duke.edu

'Division of Infectious Diseases, Duke University School of Medicine, DUMC Box 102359, Durham, NC 27710, USA

${ }^{2}$ Duke Global Health Institute, Duke University, Durham, North Carolina, USA

Full list of author information is available at the end of the article
}

C C The Author(s). 2020 Open Access This article is licensed under a Creative Commons Attribution 4.0 International License, which permits use, sharing, adaptation, distribution and reproduction in any medium or format, as long as you give appropriate credit to the original author(s) and the source, provide a link to the Creative Commons licence, and indicate if changes were made. The images or other third party material in this article are included in the article's Creative Commons licence, unless indicated otherwise in a credit line to the material. If material is not included in the article's Creative Commons licence and your intended use is not permitted by statutory regulation or exceeds the permitted use, you will need to obtain permission directly from the copyright holder. To view a copy of this licence, visit http://creativecommons.org/licenses/by/4.0/ The Creative Commons Public Domain Dedication waiver (http://creativecommons.org/publicdomain/zero/1.0/) applies to the data made available in this article, unless otherwise stated in a credit line to the data. 


\section{Background}

Despite the availability of antibiotic and antiviral therapies, advancements in vaccinations, and increased access to health care, the worldwide burden of lower respiratory tract infections (LRTI) remains immense [1], with an estimated 2.38 million deaths (all ages) attributable to LRTIs in 2016 alone [2]. Among the respiratory pathogens contributing to the high burden of LRTI morbidity and mortality are several viruses with known or suspected zoonotic transmission potential [3]. In addition to influenza viruses, these include coronaviruses $(\mathrm{CoV})$, enteroviruses (EV) [4-6] and adenoviruses [7-10]. A systematic review of the viral etiology of communityacquired pneumonia (CAP) published in 2016 found that the highest proportion of viral infection was by influenza virus, detected in $8 \%$ of cases, followed by infection by human rhinovirus (HRV), detected in $5.7 \%$ of cases. $\mathrm{AdV}$ and $\mathrm{CoV}$ were detected in $1-4 \%$ of CAP patients and $\mathrm{EV}$ were detected in $<1 \%$ [11].

Clinical diagnostics used to detect $\mathrm{CoV}, \mathrm{EV}$, and $\mathrm{AdV}$ are often designed using the gene sequences of previously identified viruses which have infected humans. Such diagnostics may miss a novel virus strain, especially such viruses which are naturally harbored in animals. Sarawak, rich in flora and fauna biodiversity [12], is located near the equator with a population that often has considerable exposure to both domestic animals and wildlife. Hence, we hypothesized that in our assessments of the etiology of pneumonia in a study population residing in an area of great animal and plant biodiversity we might be missing animal $\mathrm{CoV}, \mathrm{EV}$, and $\mathrm{AdV}$ causing human disease. In the following analyses we sought to compare real-time assay results with panspecies conventional molecular diagnostics to examine sensitivities and learn if novel viruses had been missed.

\section{Methods}

In this work we further analyzed 599 nasopharyngeal swab specimens collected during a year-long pneumonia etiology study, conducted between June 2017 and May 2018 in Sarawak, Malaysia [13]. All specimens, regardless of their real-time polymerase chain reaction (rPCR) or real-time reverse transcription polymerase chain reaction (rRT-PCR) assays results, were re-examined for evidence of $\mathrm{AdV}, \mathrm{EV}$, and $\mathrm{CoV}$ with panspecies gelbased nested PCR/RT-PCR assays. Gene sequences of all specimens found positive by the panspecies assays were then sent for sequencing and studied with the Basic Local Alignment Search Tool software (BLAST) at the National Center for Biotechnology Information (NCBI), recording all matches with greater than $80 \%$ identity. Real-time and conventional molecular assays results were then compared.

\section{Participants}

As previously described [13], study enrollment took place from June 15, 2017 to May 14, 2018 at Sibu and Kapit Hospitals in Sarawak, Malaysia. The study team relied on convenience sampling to enroll patients for the first two months of the study before enrolling patients on two out of three randomly selected days of each week, as communicated to medical officers by a study coordinator. Patients of all ages above 30 days who had been admitted to either hospital and diagnosed with pneumonia by an attending physician were considered for study eligibility. Physicians evaluated subjects for inclusion and exclusion criteria, adapted from two large and comprehensive, United States community-based pneumonia studies published in 2015 [14], and confirmed diagnosis by chest radiography within $72 \mathrm{~h}$ of hospitalization (see Supplementary Table 1). Adults 18 years of age or older provided written consent and children ages 7 to 18 provided written assent and written parental or guardian consent. For patients $<7$ years of age only parental or guardian consent was required. The study was conducted in accordance with the ethical standards of the Helsinki Declaration and received a scientific review by and ethical approval from the Malaysian Ministry of Health's Medical Research and Ethics Committee, the Duke University Health System Institutional Review Board, the Duke-NUS Medical School Ethical Review Board, and the Naval Medical Research Center-Asia Human Research Protection Program.

\section{Sampling procedures}

Consenting or assenting patients completed a brief questionnaire then permitted the collection of one nasopharyngeal (NP) swab, which was placed into a transport tube with $3 \mathrm{~mL}$ sterile viral transport medium (BD Universal Viral Transport; Becton, Dickinson and Company, Franklin Lakes, NJ) and delivered to the Sibu Hospital Clinical Research Center (SHCRC) laboratory or the Kapit Hospital laboratory where the specimen was stored at $-80^{\circ} \mathrm{C}$ until RNA or DNA extraction was performed using the QIAmp Cador Pathogen Mini Kit (Qiagen, Hilden, Germany).

\section{Real-time molecular assays for known human respiratory pathogens}

As previously reported [13], rPCR and rRT-PCR assays were initially conducted on similar BioRad CFx96 C1000 Touch Thermal Cycler Real-Time systems at SHCRC. All assays were conducted using DNA or cDNA positive controls and a nuclease-free water negative control. Cycle threshold $(\mathrm{Ct})$ values $<38$ were considered positive and $\mathrm{Ct}$ values $>40$ were considered negative. Ct values 38 to 40 were considered suspect, acknowledging that 
positivity may be the result of cross-reactivity or nonspecific amplification.

One milliliter aliquots of all specimens were shipped on dry ice then further validated at the Duke One Health Research Laboratory at Duke University in Durham, North Carolina $(n=428)$ or the Laboratory of One Health Research at Duke-NUS Medical School in Singapore $(n=171)$ where real-time assays were repeated and results were agreed upon [13].

\section{Conventional molecular assays for human or animal respiratory pathogens}

Specimens which had not been depleted from previous work were examined with new nucleic extractions and panspecies conventional PCR/RT-PCR assays at either Duke University or Duke-NUS Medical School. These panspecies assays were designed to detect both human and animal pathogens.

Viral DNA was assessed for AdV using a gel-based nested PCR assay with Invitrogen Platinum Taq DNA Polymerase Kit (Thermo Fisher Scientific, Inc., Waltham, MA) as described by Wellehan et al. (Table 1) [15]. Viral RNA was analyzed with gel-based RT-PCR assays for pan-species EV and pan-species CoV using SuperScript $^{\circ}$ III One-Step RT-PCR System with Platinum $^{\circ}$ Taq DNA Polymerase as described by Vijgen et al. and the World Health Organization [17, 18]. Specimens positive by the pan-species assays at Duke University and Duke-NUS University were sent for sequencing at Eton Bioscience, Inc., in Durham, North Carolina, and AITbiotech in Singapore, respectively. $\mathrm{CoV}$ positives were sequenced using the RT-PCR primers described in Lelli et al., 2013 [16].

Select gene sequences were then studied with the NCBI software tool BLAST using the FASTA formatted sequences and the BioEdit program (Ibis Biosciences,
Carlsbad, CA, USA). Matches with $>80 \%$ identity were reported.

The 428 specimens shipped to Duke University were additionally screened using a gel-based nested PCR assay for human AdV targeting a partial region of the hexon gene using Invitrogen Platinum Taq DNA Polymerase Kit (Thermo Fisher Scientific, Inc., Waltham, MA) [19]. We were unable to conduct the hexon assay on the specimens shipped to Duke-NUS.

\section{Statistical analysis}

Real-time assay results and baseline data from enrollment questionnaires were entered into REDCap version 7.0 before being imported into STATA version 15.0 (StataCorp, College Station, TX) to assess patient characteristics and laboratory results. Comparison of realtime and conventional assay results were conducted using STATA (Cohen's kappa coefficient) and Microsoft Excel.

\section{Results}

A total of 600 hospitalized pneumonia patients were enrolled at Sibu and Kapit Hospitals between June 15, 2017 and May 15, 2018, with 65\% of patients enrolled at Sibu Hospital. Of the enrolled subjects, 325 (54.2\%) were male. A total of 385 (64.2\%) enrolled subjects were children 5 years of age or younger and 439 (73.2\%) were of age 18 years and younger (see Supplementary Table 2).

Of the $600 \mathrm{NP}$ swabs collected, 599 (one NP was accidentally destroyed before molecular screening) were analyzed using rPCR or rRT-PCR for influenza A, B, C, and $D$ viruses, $A d V, E V, C o V$, respiratory syncytial virus subtype A (RSV-A) or RSV-B, and parainfluenza virus (types $1-4)$. Four hundred twenty-seven (427) of the total 428 samples at Duke University (one sample was depleted) were additionally analyzed by conventional PCR for

Table 1 Primer and probe sequences and gene target region for all panspecies assays. All assays were adapted from the cited references at Duke University

\begin{tabular}{|c|c|c|c|}
\hline Virus Assay & Function & Sequence & Target Gene \\
\hline \multicolumn{4}{|l|}{ Pan adenovirus [15] } \\
\hline \multirow[t]{2}{*}{ Nested PCR 1} & Forward primer (polFouter) & 5'-TNMGNGGNGGNMGNTGYTAYCC-3' & Polymerase \\
\hline & Reverse primer (polRouter) & 5'-GTDGCRAANSHNCCRTABARNGMRTT-3' & \\
\hline \multirow[t]{2}{*}{ Nested PCR 2} & Forward primer (polFinner) & 5'-GTNTWYGAYATHTGYGGHATGTAYGC-3' & \\
\hline & Reverse primer (polRinner) & 5'-CCANCCBCDRTTRTGNARNGTRA-3' & \\
\hline \multirow[t]{2}{*}{ Pan coronavirus [16] } & Forward primer & 5'-GGTTGGGACTATCCTAAGTGTGA-3' & Polymerase \\
\hline & Reverse primer & 5'-CCATCATCAGATAGAATCATCATA-3' & \\
\hline \multirow[t]{4}{*}{ Pan enterovirus [17] } & AN32 primer & 5' GTY TGC CA 3' & Capsid protein VP1 \\
\hline & AN33 primer & 5' GAY TGC CA 3' & \\
\hline & AN34 primer & $5^{\prime}$ CCR TCR TA 3' & \\
\hline & AN35 primer & 5' RCT YTG CCA 3' & \\
\hline
\end{tabular}


HRV species A, B, and C (Table 2) [20] (see Supplementary Tables $3,4,5)$.

\section{Real-time molecular assays}

A total of 62 specimens were positive by rPCR for AdV, with an overall positivity of $10.4 \%$. A total of 25 specimens were positive by rRT-PCR for EV (4.2\% positivity) and six specimens were positive by rRT-PCR for CoV (1.0\% positivity).

\section{Conventional assays}

We were surprised by the discordance between real-time and conventional molecular methods (Table 2). A total 74 specimens were positive by the panspecies AdV assay (59 at Duke University and 15 at Duke-NUS). Of the 428 specimens analyzed with the human AdV-Hexon assay, 84 specimens were positive, of which, 45 were neither positive by rPCR nor the panspecies assay. There were 44 specimens that were positive by the panspecies AdV assay that were negative by rPCR and a total 32 specimens that were positive by rPCR that were negative by the AdV panspecies assay (see Supplementary Table 6).

Forty-eight (48) of the 599 specimens across Duke $(n=24)$ and Duke-NUS $(n=24)$ were positive by panspecies enterovirus assays, nearly doubling the positivity detected by the rRT-PCR assay (see Supplementary Table 7). Of the subset of 427 specimens analyzed for HRV at Duke University, 51 (11.9\%) of the 427 specimens were positive by gel electrophoresis and sequence analysis for HRV. The positivity of the panspecies $\mathrm{CoV}$ assay was again much higher than the $1 \%$ positivity by the real-time assay, with 47 specimens positive by the panspecies $\mathrm{CoV}$ assay, 45 at Duke University and two at Duke-NUS (see Supplementary Table 8).

\section{Sequencing results}

We successfully sequenced 30 of the 74 specimens positive by the panspecies AdV assay. Of the 30 sequenced specimens, $83 \%$ were collected from pediatric patients (Table 3). The most prevalent AdV detected was human AdV type 7 (HAdV-7), with 14 total specimens having between 93 and $99 \%$ identity with HAdV-7. All 14 of the HAdV-7 specimens were collected from pediatric patients hospitalized at Sibu Hospital (Table 4). In addition to HAdV-7, sequencing revealed several human AdV type 5 (HAdV-5) detections in addition to human AdV type 4 (HAdV-41) and several AdV from the mastadenovirus genera, including the type species human mastadenovirus $C$ (Table 3 ).

The NP specimens of two pediatric patients, both enrolled on May 3, 2018 at Sibu Hospital, were found to have $100 \%$ identity with the DrAdV1/PGT0342 DNA polymerase gene, partial cds., Desmondus rotundus adenovirus 1 , an AdV previously only detected in the common vampire bat. Both specimens were positive by all three assays at Duke University. Following sequencing, the two specimens were shipped to the University of Florida for attempts at viral isolation. AdV was not detected by PCR in spent cell culture media or cells inoculated with patient specimens.

We conducted next generation sequencing (Illumina iSeq100 and Nextera DNA Flex Library Prep Kit) on the two original clinical specimens as a potential new approach for clinical laboratory diagnostics. We submitted the resultant sequence data to Chan Zuckerberg Biohub's IDseq metagenomics software pipeline [21]. However, our collaborators at IDseq found sparse DNA, not enough adenovirus-like reads to assemble a full genome. Hence, we were unable to determine with convincing rigor what might have given us the original novel conventional RT-PCR sequencing findings.

Table 2 Comparison of adenovirus (AdV), enterovirus (EV), and coronavirus (CoV) assay results across Duke and Duke-NUS analysis

\begin{tabular}{lll}
\hline AdV assays & Positive specimens (n) & Positivity (\%) \\
AdV rPCR $(n=599)$ & 62 & $10.4 \%$ \\
AdV panspecies $(n=599)$ & 74 & $12.4 \%$ \\
AdV Hex conventional $(n=428)$ & 84 & $19.6 \%$ \\
EV assays & Positive specimens (n) & Positivity (\%) \\
EV rRT-PCR $(n=599)$ & 25 & $4.2 \%$ \\
EV panspecies $(n=599)$ & 48 & $8.0 \%$ \\
HRV gel electrophoresis \& sequence analysis $(n=427)$ & 51 & $11.9 \%$ \\
CoV assays & Positive specimens (n) & Positivity (\%) \\
CoV rRT-PCR $(n=599)$ & 6 & $1.0 \%$ \\
CoV panspecies $(n=599)$ & 47 & $7.8 \%$ \\
\hline
\end{tabular}


Table 3 Results of successfully sequenced specimens positive by panspecies adenovirus [15] assay

\begin{tabular}{|c|c|c|c|c|c|c|c|}
\hline & Patient age & Hospital & Sequencing Result & $\begin{array}{l}\text { Identity Score } \\
\text { From BLAST }\end{array}$ & Accession Number from BLAST & rPCR Assay & AdV-Hexon Assay \\
\hline 1. & 6 months & Kapit & Human mastadenovirus $C$ & $98 \%$ & MH121117.1 & Neg & Neg \\
\hline 2. & 65 years & Kapit & Human mastadenovirus $\mathrm{E}$ & $99 \%$ & KY996453.1 & Pos & Pos \\
\hline 3. & 1 month & Kapit & Human mastadenovirus $C$ & $98 \%$ & MH121117.1 & Neg & Neg \\
\hline 4. & 66 years & Sibu & Human mastadenovirus $C$ & $97 \%$ & MH121097.1 & Neg & Neg \\
\hline 5. & 1 year & Sibu & Human adenovirus $C$ & $95 \%$ & MF358574.1 & Pos & $N / A^{a}$ \\
\hline 6. & 10 months & Kapit & Human adenovirus 41 & $98 \%$ & KX868523.2 & Neg & $N / A^{a}$ \\
\hline 7. & 6 months & Sibu & Human adenovirus $C$ & $98 \%$ & MF358562.1 & Pos & $N / A^{a}$ \\
\hline 8. & 4 months & Kapit & human adenovirus 41 & $98 \%$ & KX868523.2 & Neg & $N / A^{a}$ \\
\hline 9. & 1 year & Kapit & Human adenovirus 5 & $98 \%$ & MF358601.1 & Pos & Pos \\
\hline 10. & 35 years & Sibu & Human adenovirus 4 & $99 \%$ & AP014851.1 & Pos & Pos \\
\hline 11. & 3 years & Sibu & Human adenovirus B & $99 \%$ & KF268212.1 & Pos & Neg \\
\hline 12. & 2 years & Sibu & Human adenovirus 7 & $99 \%$ & MG923582.1 & Pos & Neg \\
\hline 13. & 2 years & Sibu & Human adenovirus 7 & $98 \%$ & MG923582.1 & Pos & Neg \\
\hline 14. & 9 months & Sibu & Human adenovirus 7 & $98 \%$ & MG923582.1 & Pos & Neg \\
\hline 15. & 6 months & Kapit & Human adenovirus 7 & $97 \%$ & MG923582.1 & Pos & Pos \\
\hline 16. & 2 years & Sibu & Human adenovirus 7 & $99 \%$ & MG923582.1 & Pos & $\mathrm{Neg}$ \\
\hline 17. & 1 month & Sibu & Human adenovirus 5 & $99 \%$ & MF358604.1 & Pos & Neg \\
\hline 18. & 8 months & Sibu & Human adenovirus 7 & $98 \%$ & MG923582.1 & Pos & Pos \\
\hline 19. & 8 months & Sibu & Human adenovirus 7 & $98 \%$ & MG923582.1 & Pos & Pos \\
\hline 20. & 7 months & Sibu & Human adenovirus 7 & $98 \%$ & MG923582.1 & Pos & Pos \\
\hline 21. & 1 year & Sibu & Human adenovirus 7 & $97 \%$ & MG923582.1 & Pos & Pos \\
\hline 22. & 8 months & Sibu & Desmondus rotundus adenovirus 1 & $100 \%$ & KX774303.1 & Pos & Pos \\
\hline 23. & 1 month & Sibu & Desmondus rotundus adenovirus 1 & $100 \%$ & KX774303.1 & Pos & Pos \\
\hline 24. & 6 months & Sibu & Human adenovirus 7 & $96 \%$ & MG923582.1 & Pos & Pos \\
\hline 25. & 3 months & Sibu & Human adenovirus 7 & $93 \%$ & MG923582.1 & Pos & Pos \\
\hline 26. & 4 months & Sibu & Human adenovirus 7 & $98 \%$ & MG923582.1 & Pos & Pos \\
\hline 27. & 2 years & Sibu & Human adenovirus 7 & $98 \%$ & MG923582.1 & Pos & Pos \\
\hline 28. & 2 months & Sibu & Human adenovirus 7 & $97 \%$ & MG923582.1 & Pos & Pos \\
\hline 29. & 54 years & Sibu & Human adenovirus 5 & $96 \%$ & MF358604.1 & Pos & Neg \\
\hline 30. & 78 years & Sibu & Human adenovirus 5 & $98 \%$ & MF358604.1 & Pos & Neg \\
\hline
\end{tabular}

${ }^{\mathrm{a}}$ Specimens at Duke-NUS not run by conventional AdV-Hexon assay

Of specimens found positive by the panspecies $\mathrm{CoV}$ assay at Duke University, only one was successfully sequenced. The sequenced specimen, which shared 93.7\% identity with Human CoV OC43, was negative by rRTPCR. The two positive specimens by the CoV panspecies at Duke-NUS were both sequenced with identity scores of 98.8 and $99.8 \%$ with Human CoV 229E.

Of the specimens successfully sequenced for HRV, HRV $C$ was the most prevalent species (51\%) followed by HRV A (41\%) and HRV B (8\%). Of the positive EVpanspecies specimens, 17 were successfully sequenced. Sequencing revealed three EV-71 specimens, all three collected from pediatric patients, two from Sibu Hospital and one from Kapit. There was also one EV-D68 detected and one coxsackievirus B5, also both collected from pediatric patients.

Agreement between the real-time and conventional PCR/RT-PCR assays were not as strong as we expected: adenovirus $87.2 \%$ agreement, Kappa 0.37; enterovirus $88.4 \%$ agreement, Kappa - 0.00; and coronavirus 91.8\% agreement, Kappa 0.06 (see Supplemental Tables 6, 7, 8).

\section{Discussion}

Based on positivity alone, the $\mathrm{AdV}, \mathrm{EV}$, and $\mathrm{CoV}$ panspecies assays found in total an additional 135 positive detections that were not found positive by real-time PCR. Only two specimens were positive by both the conventional and real-time $\mathrm{CoV}$ assays, with an additional 
Table 4 Human Adenovirus 7 Infections Detected at Sibu

\begin{tabular}{llll} 
Hospital & & & \\
\hline Gender & Age (months) & Specimen Collection & Coinfection \\
\hline Male & 20 & $2 / 6 / 18$ & \\
Female & 25 & $2 / 9 / 18$ & RSV-B \\
Male & 9 & $3 / 7 / 18$ & \\
Male & 25 & $3 / 22 / 18$ & \\
Female & 9 & $4 / 27 / 18$ & RSV-B \\
Male & 8 & $4 / 30 / 18$ & \\
Female & 7 & $4 / 30 / 18$ & \\
Female & 16 & $4 / 30 / 18$ & RSV-B \\
Male & 6 & $5 / 4 / 18$ & EV \\
Female & 3 & $5 / 4 / 18$ & \\
Male & 4 & $5 / 4 / 18$ & RSV-B \\
Male & 20 & $5 / 4 / 18$ & RSV-A \\
Female & 3 & $5 / 7 / 18$ & \\
Male & 7 & $3 / 7 / 18$ & \\
\hline & & &
\end{tabular}

45 detections by the panspecies $\mathrm{CoV}$ assay, including a specimen that was successfully sequenced with $93.7 \%$ identity with CoV OC43 (see Supplementary Tables 5, 8). Similarly, only two of the specimens were positive by both the conventional and real-time EV assays, with 48 additional detections by the panspecies EV assay (see Supplementary Table 4,7). These findings would suggest that where possible, it is advisable to supplement realtime assay results with conventional molecular methods; however, we recognize the challenges to running conventional assays as compared to real-time.

The discrepancies between the AdV assays were greater with the AdV-Hex conventional assay than the rPCR assay. Comparing the results of the 30 successfully sequenced specimens positive by the panspecies assay, the rPCR assay detected 26 of the 30 positive specimens (84\% agreement) (Table 3) while the conventional AdvHexon assay (only run on 26 of the 30 specimens) detected 15 of the positive specimens (53\% agreement).

The 51 specimens positive by gel electrophoresis and sequence analysis for HRV were not the same as the 48 specimens positive by panspecies enterovirus assays (see Supplementary Table 4); however, we recognize the potential for cross-reactivity of the enterovirus primers as a potential limitation of the study. Additionally, during sequencing, $13 \mathrm{AdV}$ specimens and two CoV specimens cross amplified human nucleic acid (see Supplementary Tables 3, 5).

As real-time PCR and RT-PCR have become gold standards in molecular diagnostics, conventional molecular methods are used less frequently due largely to their added complexity and time requirements. In a comparative study of real-time PCR versus other conventional detection methods such as cultures, urine antigen assays, and serologic tests, Yoshii et al. found that among adult community-acquired pneumonia patients, real-time PCR was significantly better able to detect both viral and bacterial pathogens in NP and sputum specimens compared to other conventional methods (72\% pathogen detection rate versus 57\%) [22]. However, had we relied exclusively on the rPCR and rRT-PCR results to select which specimens to sequence, we would have had an incomplete understanding of the epidemiology of these three viruses contributing to the burden of LRTIs in these two hospitals. The discordance detected in the results supports the decision to conduct both real-time and conventional PCR on these specimens, as these findings complemented one another.

This study had a number of limitations. Patient selection was dependent upon the willingness of clinicians to refer their patients for study, and the willingness of patients to enroll. Hence, the participants should be viewed as a convenience sample. Some of the differences in detection between molecular methods may be attributed to differences in the analytic sensitivities of the molecular methods. Our study was limited to a panel of viruses and therefore we were unable to determine the prevalence of bacterial mono- or coinfections within the population. We were not able to ascertain virus viability in the remote setting (Sarawak, Malaysia) as no virus culture capability was available. Because of multiple freeze thaw cycles, we only pursued live virus rescue for the two specimens with molecular evidence of a novel bat-like adenovirus. As described, those culture efforts failed. Hence, we were uncertain which of our molecular detections reflect live virus infections. A number of the samples detected positive by conventional assays were not successfully sequenced, potentially because the amount of starting material was suboptimal. Additionally, while our two laboratory teams (Singapore and USA) closely followed the same written procedures, there might have been differences in technique that contributed to inter-laboratory ascertainment biases. Even with these limitations it seems clear that the panspecies approaches for these three virus types were a more sensitive method.

\section{Conclusions}

Through the panspecies conventional PCR/RT-PCR assays, we were able to detect LRTI that may have otherwise been missed with the rPCR/rRT-PCR assays. This was especially true for adenoviruses, with 44 specimens positive by the conventional HAdV panspecies assay that were negative by rPCR, which we would otherwise have been missed. Two specimens sharing 97\% and 98\% identity with human mastadenovirus C were negative by rPCR but positive by the panspecies assay and successfully sequenced. Another specimen, 
sharing $98 \%$ identity with human HAdV-41 was successfully sequenced after a positive panspecies assay result but was negative by rPCR. Hence, we conclude that where a sensitivity problem may be suspected, real-time assay results might be supplemented with conventional molecular methods.

\section{Supplementary information}

Supplementary information accompanies this paper at https://doi.org/10 1186/s40794-020-00114-2.

Additional file 1: Table S1. Inclusion and exclusion criteria checklist used by medical officers while assessing patients for enrollment eligibility. Table S2. Demographic characteristics and exposure variables reported upon enrollment among patients hospitalized with pneumonia at Sibu and Kapit hospitals, between June 2017 and May 2018. Table S3. All specimens positive by one or more molecular method demonstrating discrepancies across adenovirus (AdV) assays. Table S4. All specimens positive by one or more molecular method demonstrating discrepancies across enterovirus (EV) assays. Table S5. All specimens positive by one or more molecular method demonstrating discrepancies across coronavirus (CoV) assays. Table S6. Comparison of positivity of panspecies conventional PCR versus real-time PCR for AdV. Table S7. Comparison of positivity of panspecies conventional PCR versus real-time PCR for EV. Table S8. Comparison of positivity of panspecies conventional PCR versus real-time PCR for CoV.

\section{Abbreviations}

AdV: Adenovirus; BLAST: Basic Local Alignment Search Tool software; CoV: Coronavirus; EV: Enterovirus; HRV: Human rhinovirus; LRTI: Lower respiratory tract infections; NCBI: National Center for Biotechnology Information; NP: Nasopharyngeal; PCR: Polymerase chain reaction; RSV: Respiratory syncytial virus; rPCR: Real-time polymerase chain reaction; rRT-PCR: Real-time reverse-transcription polymerase chain reaction; SHCR C: Sibu Hospital Clinical Research Center

\section{Acknowledgements}

We thank the Director General of Health, Malaysia for his permission to publish this paper. This work was conducted in partnership with Duke University, the Duke Global Health Institute, Sibu Hospital Clinical Research Center and SEGi University Sibu Clinical Campus. We thank the Medical Officers of Sibu and Kapit Hospitals (namely Sing-Ling Kong, Nga-Hung Ngu, Khai-Fatt Chao, Cheng-Ing Kong, Zhen-Hao Chin, Edmund Kwang-Yuen Wong, Tiana Ti, and Hilary Hon-Yun Kueh) for enrolling patients. We thank the laboratory staff at Kapit Hospital (namely Cornelius Jambol, Goh Hieng Hua, and Velarie Bill) and Tiing-Tiing Chua at Sibu Hospital for processing specimens. We thank Joe DeRisi and Vida Ahyong with Chan Zuckerberg Biohub's IDseq for their support with the sequencing data. We thank Dr. Cheesan Lee for helping us to begin this collaboration. We thank Sarah Paust, Calvin Wang, Christine Wang, and Anfal Abdelgadir for laboratory support.

\section{Authors' contributions}

JF, THT, JT, KCH, KM, AB, SCW, TMW and GG collected samples and data. THT, $\mathrm{KCH}, \mathrm{SCW}$ and TMW facilitated the study in the hospitals. EB, JL, KM, JT, AB, $\mathrm{TN}, \mathrm{DD}$, and ST processed and analyzed samples. JF, EB, and GG wrote the manuscript. $J$, PB, and GG provided technical expertise and guided manuscript development. GG provided in country support and guided the student led team. All the authors reviewed the final version of the manuscript and agreed to its submission.

\section{Funding}

This work was supported by the US Naval Medical Research Center-Asia and Vysnova Partners [SC-2016-SABER-003-002, SC-2017-SABER-010-001]; and by Professor Gregory Gray's discretionary funds from Duke University's Global Health Institute.

\section{Availability of data and materials}

The data collected during the current study are available from the corresponding author on reasonable request.

\section{Ethics approval and consent to participate}

The study was conducted in accordance with the ethical standards of the Helsinki Declaration and received a scientific review by and ethical approval from the Malaysian Ministry of Health's Medical Research and Ethics Committee, the Duke University Health System Institutional Review Board, the Duke-NUS Medical School Ethical Review Board, and the Naval Medical Research Center-Asia Human Research Protection Program.

\section{Consent for publication}

Not applicable.

\section{Competing interests}

The authors declare no conflict of interest.

\section{Author details}

'Division of Infectious Diseases, Duke University School of Medicine, DUMC Box 102359, Durham, NC 27710, USA. ²Duke Global Health Institute, Duke University, Durham, North Carolina, USA. ${ }^{3}$ Institute for Global Health Sciences, University of California, San Francisco, California, USA. ${ }^{4}$ Department of Public Health, Texas Tech University Health Sciences Center, Abilene, TX, USA.

${ }^{5}$ Clinical Research Center, Sibu Hospital, Ministry of Health Malaysia, Sibu, Sarawak, Malaysia. ${ }^{6}$ Faculty of Medicine, SEGi University, Kota Damansara, Selangor, Malaysia. ${ }^{7}$ Department of Paediatrics, Sibu Hospital, Ministry of Health Malaysia, Sibu, Sarawak, Malaysia. ${ }^{8}$ Kapit Hospital, Ministry of Health Malaysia, Kapit, Sarawak, Malaysia. ${ }^{9}$ Department of Environmental and Global Health, University of Florida, Gainesville, Florida, USA. ${ }^{10}$ Emerging Pathogens Institute, University of Florida, Gainesville, Florida, USA. ${ }^{11}$ Emerging Infectious Disease Program, Duke-NUS Medical School, Singapore, Singapore.

${ }^{12}$ Department of Medicine, Sibu Hospital, Ministry of Health Malaysia, Sibu, Sarawak, Malaysia. ${ }^{13}$ Naval Medical Research Center-Asia, Singapore,

Singapore. ${ }^{14} \mathrm{Global}$ Health Center, Duke Kunshan University, Kunshan, China.

Received: 5 April 2020 Accepted: 27 July 2020

Published online: 12 August 2020

\section{References}

1. Murdoch DR, Howie SRC. The global burden of lower respiratory infections: making progress, but we need to do better. Lancet Infect Dis. 2018;18(11): 1162-3.

2. Troeger $\mathrm{C}$, et al. Estimates of the global, regional, and national morbidity, mortality, and aetiologies of lower respiratory infections in 195 countries, 1990-2016: a systematic analysis for the Global Burden of Disease Study 2016. Lancet Infect Dis. 2018;18(11):1191-210.

3. Bailey ES, et al. A mini review of the zoonotic threat potential of influenza viruses, coronaviruses, adenoviruses, and enteroviruses. Front Public Health. 2018;6:104.

4. Fieldhouse JK, et al. A systematic review of evidence that enteroviruses may be zoonotic. Emerg Microbes Infect. 2018;7(1):164

5. Gür S, Yapkiç O, Yilmaz A. Serological survey of bovine enterovirus type 1 in different mammalian species in Turkey. Zoonoses Public Health. 2008;55(2): 106-11.

6. Grützmacher KS, et al. Codetection of respiratory syncytial virus in habituated wild Western lowland gorillas and humans during a respiratory disease outbreak. EcoHealth. 2016:13(3):499-510.

7. Chen EC, et al. Cross-Species Transmission of a Novel Adenovirus Associated with a Fulminant Pneumonia Outbreak in a New World Monkey Colony. PLoS Pathog. 2011;7(7):e1002155.

8. Chiu $C Y$, et al. A novel adenovirus species associated with an acute respiratory outbreak in a baboon colony and evidence of coincident human infection. MBio. 2013;4(2):e00084

9. Phan TG, et al. Human adenovirus type 1 related to feline adenovirus: evidence of interspecies transmission. Clin Lab. 2006;52(9-10):515-8.

10. Borkenhagen LK, et al. Are adenoviruses zoonotic? A systematic review of the evidence. Emerg Microbes Infect. 2019;8(1):1679-87.

11. Burk $M$, et al. Viral infection in community-acquired pneumonia: systematic review and meta-analysis. Eur Respir Rev. 2016;25(140):178. 
12. Primack RB, Hall P. Biodiversity and Forest change in Malaysian Borneo. BioScience. 1992;42(11):829-37.

13. Toh T-H, et al. High prevalence of viral infections among hospitalized pneumonia patients in equatorial Sarawak, Malaysia. Open Forum Infect Dis. 2019;6(3):ofz074.

14. Jain $\mathrm{S}$, et al. Community-acquired pneumonia requiring hospitalization among U.S. children. N Engl J Med. 2015;372(9):835-45.

15. Wellehan JF, et al. Detection and analysis of six lizard adenoviruses by consensus primer PCR provides further evidence of a reptilian origin for the atadenoviruses. J Virol. 2004;78(23):13366-9.

16. Lelli $\mathrm{D}$, et al. Detection of coronaviruses in bats of various species in Italy. Viruses. 2013;5(11):2679-89.

17. World Health Organization (WHO) Regional Office for Europe. Enterovirus surveillance guidelines: Guidelines for enterovirus surveillance in support of the Polio Eradication Initiate. Copenhagen: WHO; 2015. Available from: http://www.euro.who.int/_data/assets/pdf_file/0020/272810/ EnterovirusSurveillanceGuidelines.pdf.

18. Vijgen $L$, et al. A pancoronavirus RT-PCR assay for detection of all known coronaviruses. Methods Mol Biol. 2008;454:3-12.

19. Lu X, Erdman DD. Molecular typing of human adenoviruses by PCR and sequencing of a partial region of the hexon gene. Arch Virol. 2006;151(8): 1587-602.

20. Savolainen C, et al. Genetic clustering of all 102 human rhinovirus prototype strains: serotype 87 is close to human enterovirus 70. J Gen Virol. 2002;83(2): 333-40.

21. Saha S, et al. Unbiased metagenomic sequencing for pediatric meningitis in Bangladesh reveals neuroinvasive Chikungunya virus outbreak and other unrealized pathogens. mBio. 2019;10(6):e02877-19.

22. Yoshii Y, et al. Identification of pathogens by comprehensive real-time PCR versus conventional methods in community-acquired pneumonia in Japanese adults. Infect Dis (Lond). 2016;48(11-12):782-8.

\section{Publisher's Note}

Springer Nature remains neutral with regard to jurisdictional claims in published maps and institutional affiliations.

Ready to submit your research? Choose BMC and benefit from:

- fast, convenient online submission

- thorough peer review by experienced researchers in your field

- rapid publication on acceptance

- support for research data, including large and complex data types

- gold Open Access which fosters wider collaboration and increased citations

- maximum visibility for your research: over $100 \mathrm{M}$ website views per year

At $\mathrm{BMC}$, research is always in progress.

Learn more biomedcentral.com/submissions 